\title{
Slow conduction and gap junction remodeling in murine ventricle after chronic alcohol ingestion
}

\author{
Yu-Jun Lai', Chung-Lieh Hung ${ }^{1}$, Ray-Ching Hong ${ }^{1}$, Ya-Ming Tseng ${ }^{1}$, Cheng-l Lin², Yu-Shien Ko ${ }^{3}$, Cheng-Ho Tsai ${ }^{1}$ \\ and Hung-l Yeh ${ }^{1 *}$
}

\begin{abstract}
Background: Long-term heavy alcohol drinkers are prone to the development of cardiac arrhythmia. To understand the mechanisms, we evaluated the cardiac structural and electrophysiological changes in mice chronically drinking excessive alcohol.
\end{abstract}

Results: Male C57BL/6J mice were given 36\% alcohol in the drinking water. Those given blank water were used as control. Twelve weeks later, the phenotypic characteristics of the heart, including gap junctions and electrical properties were examined. In the alcohol group the ventricles contained a smaller size of cardiomyocytes and a higher density of capillary networks, compared to the control. Western blots showed that, after drinking alcohol, the content of connexin43 (Cx43) protein in the left ventricle was increased by $18 \%(p<0.05)$. Consistently, immunoconfocal microscopy demonstrated that $\mathrm{C} \times 43$ gap junctions were up-regulated in the alcohol group with a disorganized distribution, compared to the control. Optical mapping showed that the alcohol group had a reduced conduction velocity ( $40 \pm 18$ vs $60 \pm 7 \mathrm{~cm} / \mathrm{sec}, \mathrm{p}<0.05$ ) and a higher incidence of ventricular tachyarrhythmia (62\% vs $30 \%, p<0.05)$.

Conclusion: Long-term excessive alcohol intake resulted in extensive cardiac remodeling, including changes in expression and distribution of gap junctions, growth of capillary network, reduction of cardiomyocyte size, and decrease of myocardial conduction.

Keywords: alcohol, arrhythmia, remodeling, gap junctions, optical mapping

\section{Background}

Excessive alcohol ingestion is harmful to the heart [1-4]. Previous studies have shown that manifestations of alcoholic cardiac suppression include mechanical dysfunction and electrical instability [5-7]. Physiologically, effective pumping of the heart requires coordination of contraction between individual cardiomyocytes, which depends mainly on the proper propagation of action potentials. Disturbance in the spread of action potential along the myocardium also plays a key role in the formation of cardiac arrhythmia. At the subcellular level, transmission of action potential between adjacent cardiomyocytes goes through gap junctions $[8,9]$.

\footnotetext{
* Correspondence: hiyeh@ms1.mmh.org.tw

'Departments of Internal Medicine and Medical Research, Mackay Memorial Hospital, Mackay Medicine, Nursing and Management College, Mackay Medical College, New Taipei City, Taiwan

Full list of author information is available at the end of the article
}

Gap junctions, composed of molecules belonging to the connexin multi-gene family, are clusters of cell membrane protein channels, which in the ventricular working cardiomyocytes are mainly made of connexin $43(\mathrm{Cx} 43)$ $[8,9]$. Change of the expression patterns of the connexins has been demonstrated to be associated with mechanical dysfunction and contributes to the development of cardiac arrhythmia $[10,11]$. However, the effect of alcohol on the expression of cardiac connexins remained unclear. To this end we in this study examined the ventricular myocardium, including the morphology, the gap junction distribution, and connexins expression as well as the electrophysiological properties, in mice after 12-week intake of $36 \%$ alcohol as the only source of fluid. A previous study giving mice high concentration of ethanol in the drinking water showed that the blood level of ethanol in the animals reached the level affecting physiology and/ or behavior [12]. 


\section{Results}

Weight change, alcohol levels, and histological examination

Initially the two groups of animals had similar weights (alcohol, $20.7 \pm 0.2$ g; control, $20.9 \pm 0.2$ g). However, one week later, in contrast to the weight gain in the control group ( $23.7 \pm 0.2 \mathrm{~g}$ ), the alcohol group decreased slightly in weight $(20.1 \pm 0.3 \mathrm{~g})$. Thereafter both groups gained weight gradually and throughout the remaining experiment period the alcohol group was lighter than the control group (at the end of the experiment, $26.0 \pm 1$ vs $29.4 \pm 0.8 \mathrm{~g}, \mathrm{p}<0.01)$. Similarly, at the end of the experiment the heart was lighter in the alcohol group (112 \pm 3 vs $131 \pm 3 \mathrm{mg}, \mathrm{p}<0.01)$. Comparison of the ratio of heart weight to body weight showed that both groups had a similar ratio (alcohol $0.44 \pm 0.01 \%$, control $0.45 \pm$ $0.01 \%)$. Alcohol was detected in the serum of the alcohol group $(125 \pm 13 \mathrm{mg} / \mathrm{dl})$, but not detectable in the control group.

Histological examination showed a remarkable remodeling of ventricular cardiomyocytes after 12 weeks of alcohol drinking. In samples stained with WGA the cell borders were clearly seen (Figure 1). Analysis of cardiomyocytes lying horizontally in the sections showed that the cells were smaller in the alcohol group, and the difference of size was seen across the whole layer of the ventricular wall (length, epicardial, $86 \pm 2$ vs $105 \pm 2 \mu \mathrm{m}$; endocardial $96 \pm 3$ vs $114 \pm 4 \mu \mathrm{m}$; width, epicardial, $22 \pm$ 1 vs $26 \pm 1 \mu \mathrm{m}$; endocardial, $20 \pm 1$ vs $23 \pm 1 \mu \mathrm{m}$; area, epicardial, $1.9 \pm 0.1$ vs $2.7 \pm 0.1 \times 10^{3} \mu \mathrm{m}^{2}$; endocardial, $1.9 \pm 0.1$ vs $2.6 \pm 0.1 \times 10^{3} \mu \mathrm{m}^{2}$; all $\mathrm{p}<0.05$, see Figure $1 \mathrm{~A}$ through 1D). Consistently, the number of cell nucleus per unit area of cardiac muscle was increased in the alcohol group $\left(3.2 \pm 0.1\right.$ vs $3.9 \pm 0.1$ nuclei $\times 10^{3} / \mathrm{mm}^{2}, \mathrm{p}<$ 0.05 , see Figure $2 \mathrm{~A}, \mathrm{~B}$, and left lower histogram). Apart from the change of the cardiomyocytes, a higher density of capillary network was seen in the alcohol group (3.6 \pm 1.4 vs $2.5 \pm 1.3$ capillaries $\times 10^{3} / \mathrm{mm}^{2}, \mathrm{p}<0.05$, Figure $2 \mathrm{C}, \mathrm{D}$, and right lower histogram). Similarly, development of fibrosis was rarely found in the two groups.

\section{Immunodetection of Cx43 gap junctions}

Western blotting showed that the content of $\mathrm{Cx} 43$ protein in the left ventricle of the alcohol group was $18 \%$ more compared to the control ( $<<0.05$, see Figure 3 ). Such an up-regulation of $\mathrm{Cx} 43$ was also confirmed by immunoconfocal microscopy (Figure 1E through $1 \mathrm{H}$ ), which, after image analysis, showed that in the alcohol
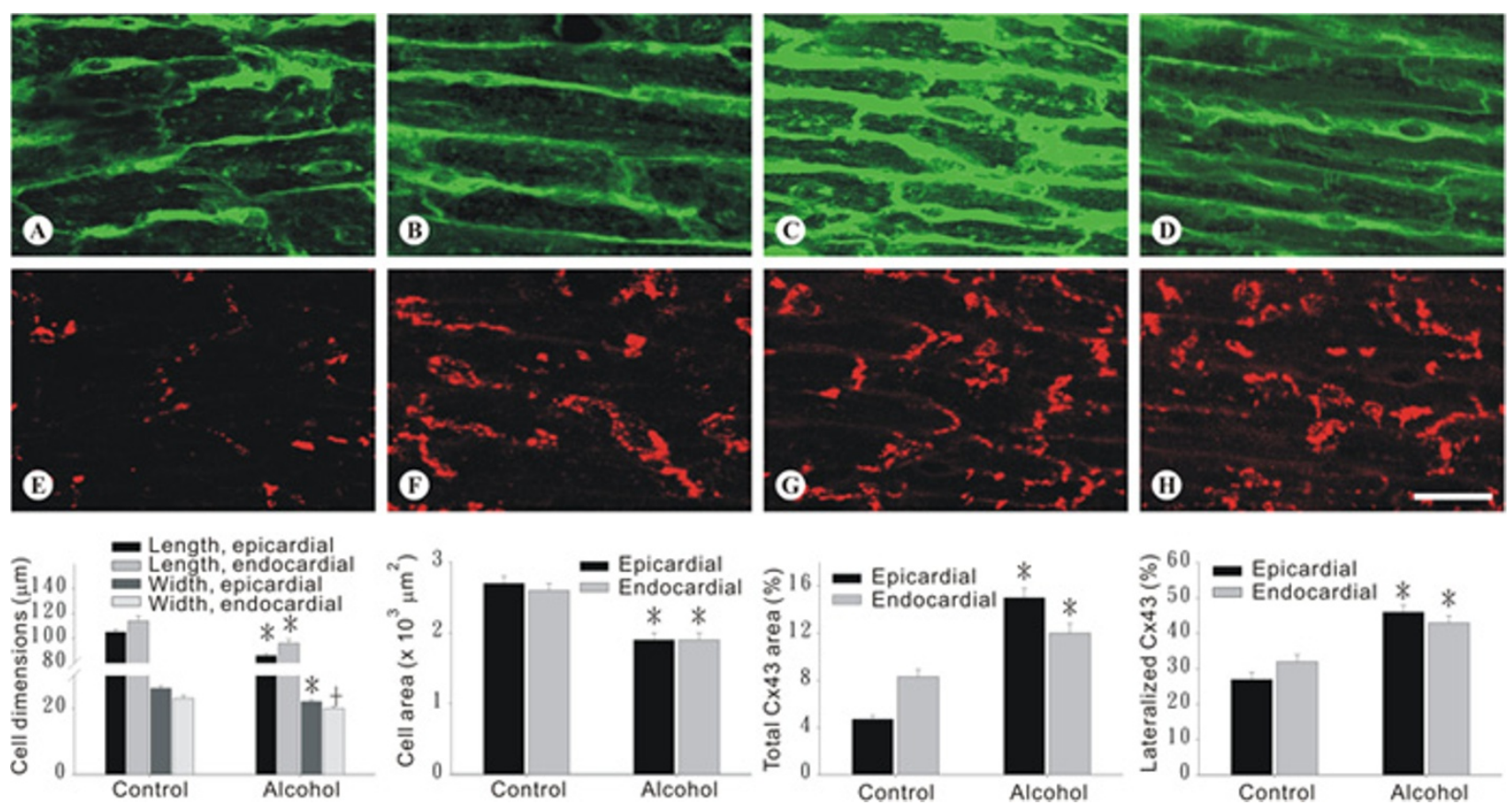

Figure 1 Reduced cardiomyocyte size and disturbed gap junction distribution in the left myocardium of mice with long-term alcohol ingestion. Immunocofocal images of cardiac muscle double stained for WGA (A through D) and CX43 (E through H). Images in each column are of the same field. Compared to the control group ( $A$ and B) the cardiomyocytes in the alcohol group (C and D) are smaller in size, but contain more Cx43 gap junctions, as shown in the below histograms, each of which represents more than 80 randomly selected cells from three animals of each group. A and C are taken from the epicardial side, B and D from the endocardial side of the left ventricular free wall. Note that the labels of WGA are more prominent in the alcohol group. In each histograms * and + respectively indicate $p<0.01$ and $p<0.05$ compared to the corresponding bar of the control group. See text for details. All images are of the same magnification. Bar, $30 \mu \mathrm{m}$. 

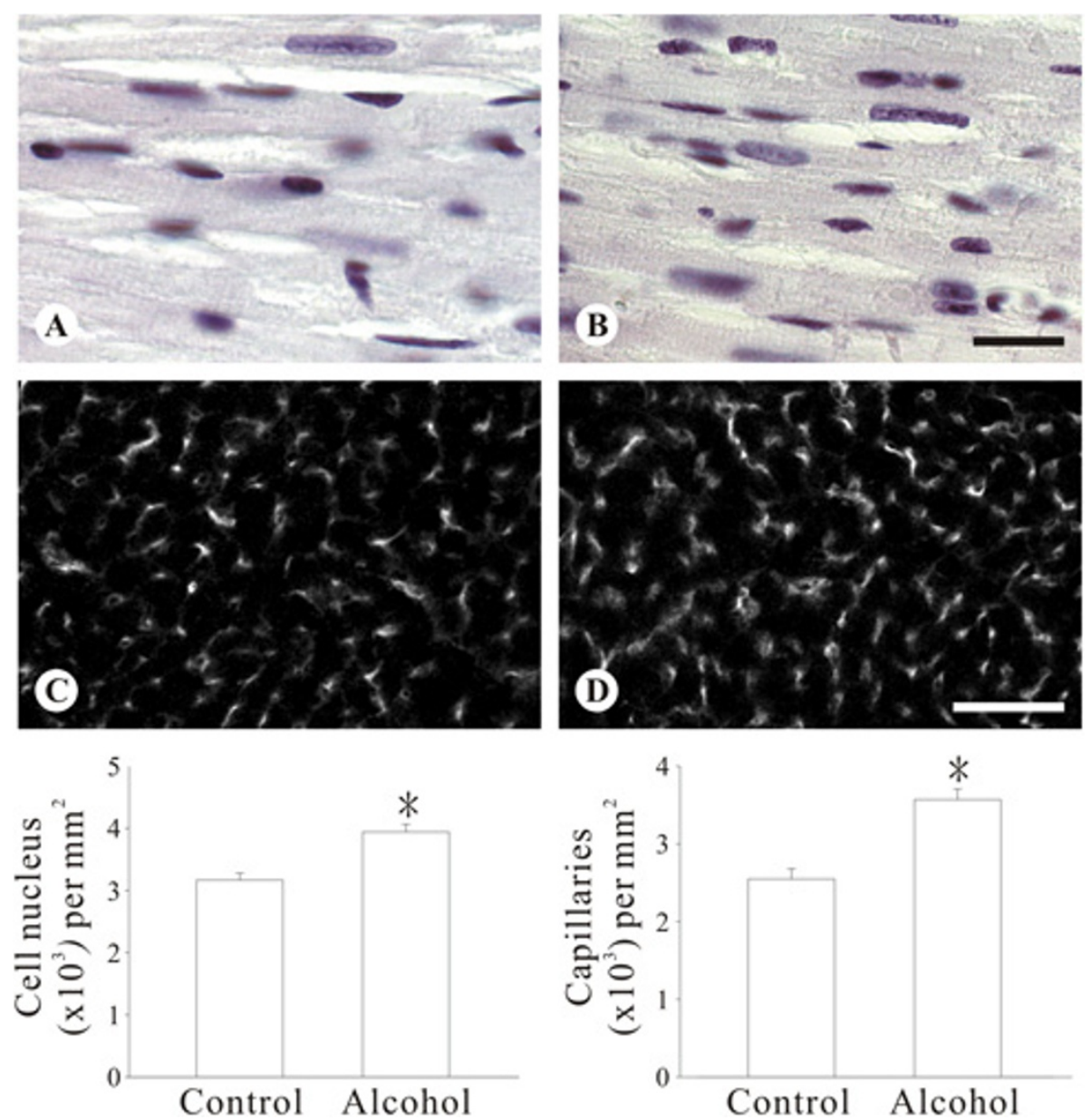

Figure 2 Increased cellularity number and capillary network in the left ventricular myocardium of mice with long-term alcohol ingestion. Cellularity ( $A$ and $B$, stained with hematoxyllin) and capillary network ( $C$ and D, stained for CD31) in the left ventricular myocardium. $A$ and $C$, are from the control group and B and D from the alcohol group. Results of analysis are shown in the histograms. In each histogram $(n=4$ or 5$)$ * indicate $p<0.05$ compared to the control group. See text for details. Images $A$ and $B$ are of the same magnification; $C$ and $D$ are of the same magnification. Bar in B, $20 \mu \mathrm{m}$; in $\mathrm{D}, 60 \mu \mathrm{m}$.

group the amount of Cx43 gap junction area per cell was increased by $219.1 \%(\mathrm{p}<0.01)$ in the epicardial portion and $44.6 \%(\mathrm{p}<0.01)$ in the endocardial portion of the myocardium. In addition, the distribution of $\mathrm{Cx} 43$ gap junctions was altered. In the control group, $\mathrm{Cx} 43$ gap junctions were mainly confined to the intercalated disk area (Figure 1E and 1F). However, in mice drinking alcohol, a substantial amount of the gap junctions were present along the lateral border of the cardiomyocytes (epicardial, $46 \pm 2$ vs $27 \pm 2 \%$; endocardial, $43 \pm 2$ vs $32 \pm$ $2 \%$, both $\mathrm{p}<0.05$, see Figure $1 \mathrm{G}$ and $1 \mathrm{H}$ ).

\section{Electron microscopy}

In the control group, cardiomyocytes were intimately packed together (Figure 4A). In contrast, in the alcohol group individual cardiomyocytes were loosely packed, owing to an increased number of capillaries as well as loose pericapillary spaces (Figure 4B). In addition to the extracellular difference, inside the cardiomyocytes swollen mitochondria with disrupted cistern were not infrequently seen in the alcohol group (Figure 4D), but rarely seen in the control (Figure 4C and 4E). Gap junctions were frequently seen in the vicinity of the adherens junctions for both groups (Figure 4E and 4F).

\section{Optical mapping}

During optical mapping examination ventricular tachyarrhythmia, including ventricular tachycardia and ventricular fibrillation, was frequently found in the alcohol group. Therefore, we compared the incidence of ventricular tachyarrhythmia in 29 mice of each group. As shown in the upper left histogram of Figure 5, 18 (62\%) mice of the alcohol group had ventricular tachyarrhythmia, in contrast to $9(31 \%)$ mice in the control 


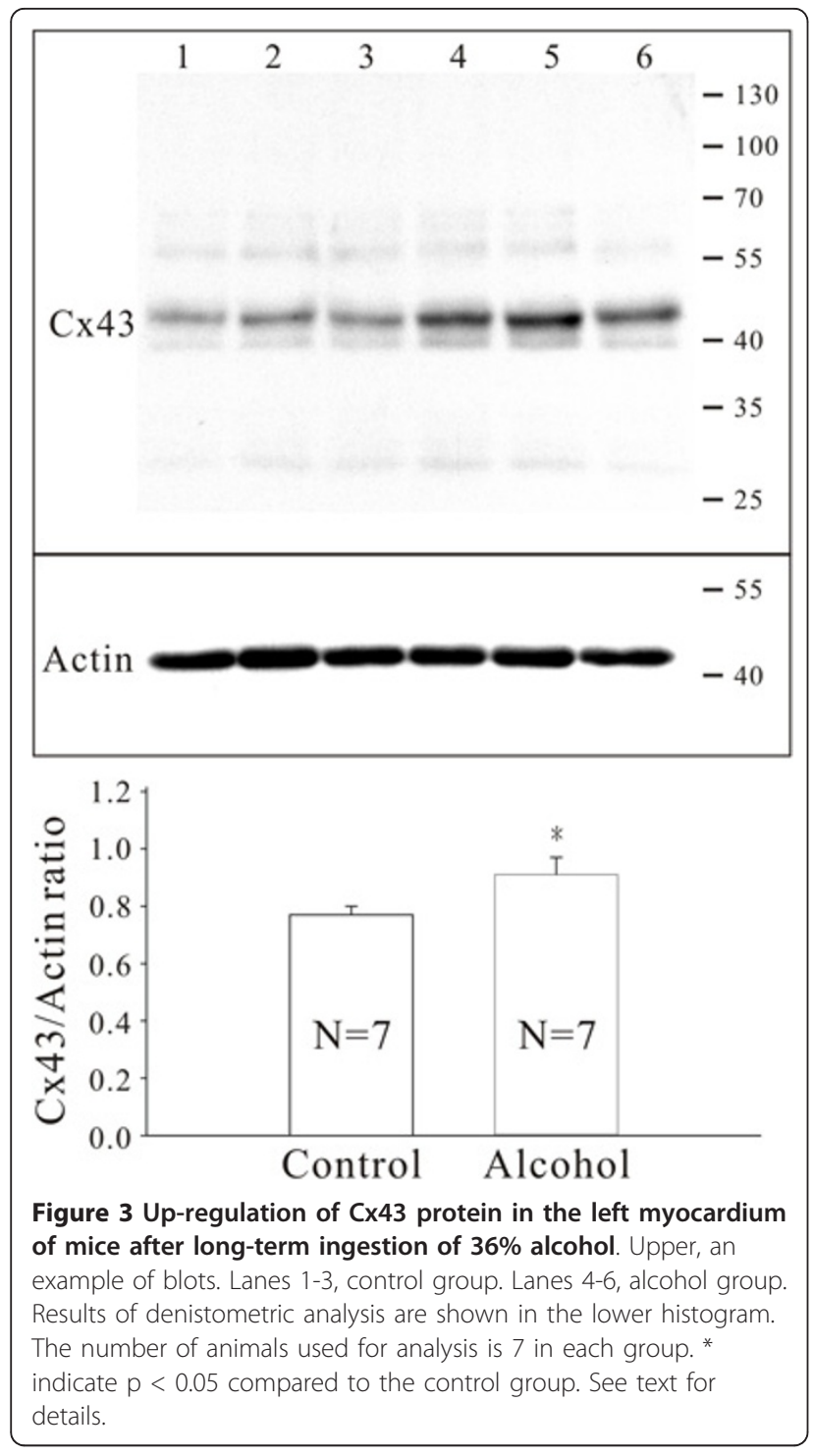

group ( $\mathrm{p}<0.05)$. In the remaining 11 mice of the alcohol group, 9 mice remained drivenable throughout the 50 minutes recording period. Conduction velocity, derived from activation map (Figure 5, lower panel), was calculated from the 9 mice of the alcohol group and compared to the initial 9 mice drivenable throughout the recording period of the control group. The result showed that the conduction velocity was significantly slower in the alcohol group (for each time point measured, all $\mathrm{p}<0.05$, see Figure $5 \mathrm{~A}, \mathrm{~B}$, and upper right histogram). In addition, in the activation map of the pacemaker-drivenable hearts the direction of activation in the control group was all from the left upper toward the right lower throughout the examination (Figure 5B). In contrast, conduction disturbance was commonly seen in the alcohol group (Figure 5C).

\section{Discussion}

This study showed that, after ingesting 36\% alcohol as the only source of fluid for 12 weeks, the murine ventricle underwent a remarkable remodeling, including reduction of cardiomyocyte size, increase in capillary network, up-regulation and lateralization of gap junctions, and slow down of conduction. In addition, animals in the alcohol group were more vulnerable to the development of ventricular tachyarrhythmia during optical mapping examination. These findings are complementary to the current knowledge of alcoholic heart disease.

In humans, long-term heavy alcohol consumption induces heart failure, the pathological findings of which are characterized by a form of non-ischemic, dilated cardiomyopathy, specifically called alcoholic cardiomyopathy [13]. Clinical studies of people regularly consuming large amount of alcohol have shown that before the development of heart failure the left ventricle volume increases and the ventricular wall thickness also increases $[14,15]$. Several controlled clinical studies have shown a dosedependent depressor effect of alcohol on LV function, an effect that progressively induces the development of lowoutput dilated cardiomyopathy leading to congestive heart failure and sudden death [13,16,17]. However, information of the individual cardiomyocytes at an earlier stage before the heart is enlarged remained deficient. In the present study, compared to the control animals, the alcoholic animals had smaller cardiomyocytes, which is consistent with the grossly lighter hearts. The change of cell size is opposite to the finding from experiments feeding mice an alcohol-containing liquid diet, in which hypertrophy of cardiomyocytes was reported [18]. Chronic alcohol exposure had been reported to lead to a loss of myofibrillar protein [19], which may explain the reduction in cell size found in the present study. On the other hand, the absence of cardiac fibrosis in the present study is consistent with the previous report [18].

The increase in cell nucleus number per unit area in the present study is consistent with the smaller size of individual cardiomyocytes. However, the increase of nucleus is also attributable to the growth of capillary network, a novel finding of the present study. Previous studies in humans and rabbits had demonstrated that alcohol changed the morphology of capillary endothelial cells in the heart $[20,21]$. To our knowledge the growth of capillary network in the heart after chronic excessive alcohol consumption had not been reported. Regarding the mechanism of the growth, although moderate levels of ethanol was shown to induce expression of vascular endothelial growth factor and stimulate angiogenesis [22], chronic ingestion of ethanol in rats was reported to increase the ventricular expression of p53 [23], which is known to inhibit angiogenesis [24]. Exploration of the mechanisms 

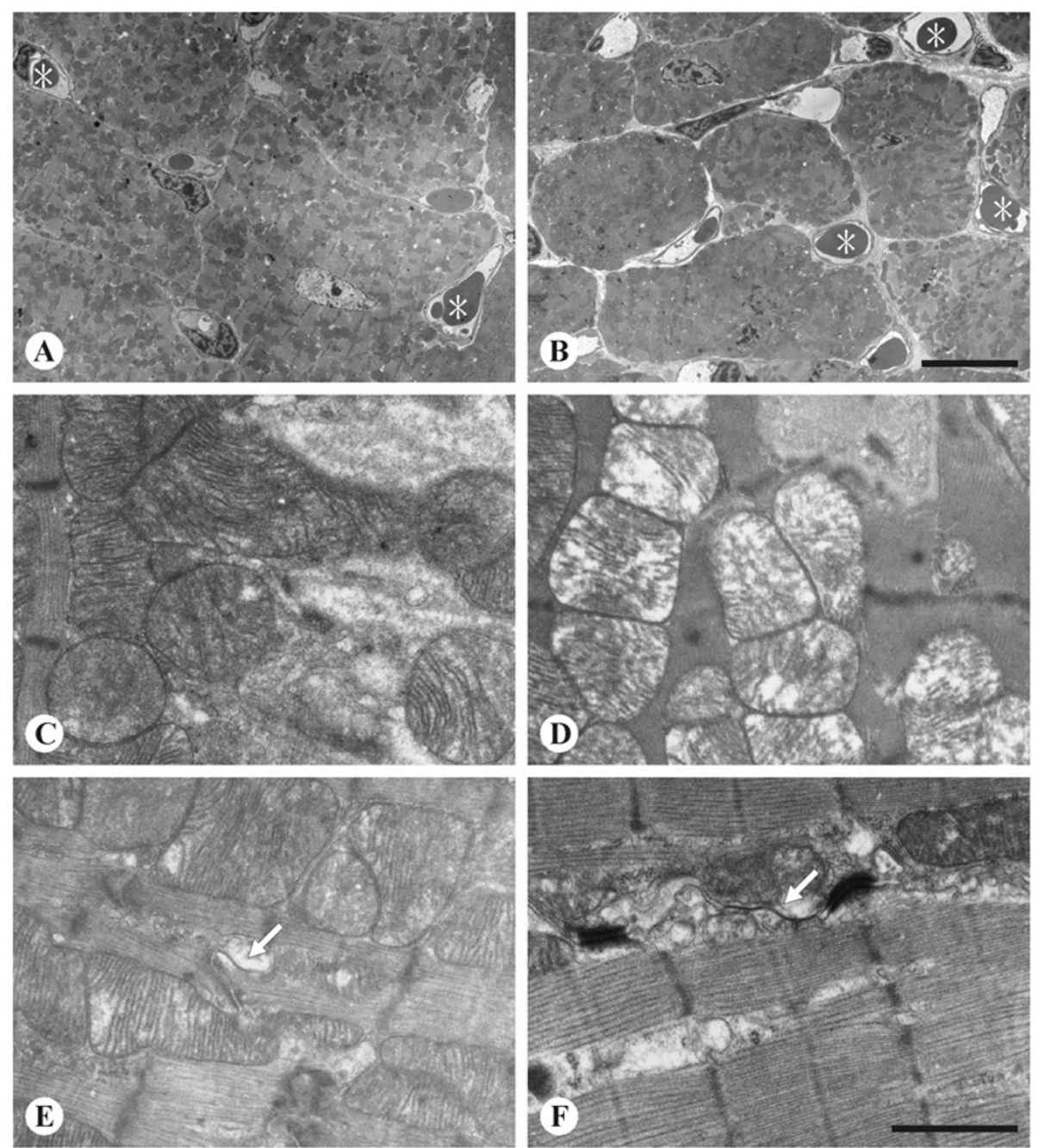

Figure 4 Ultrastructural examination of intercardiomyocyte space, mitochondria, and gap junctions. Ultrastructural examination of intercardiomyocyte space ( $A$ and $B$ ), mitochondria ( $C$ and $D)$, and gap junctions ( $E$ and $F$ ). $A, C$, and $E$ are from the control mice and $B, D$, and $F$ from the alcoholic mice. See text for details. Note that compared to the control (A), in mice drinking alcohol (B) not only capillaries with loose pericapillary spaces $\left(^{*}\right)$ are more frequently seen, but the individual cardiomyocytes are smaller. Arrows in $\mathrm{E}$ and $\mathrm{F}$ indicate gap junctions. Images $A$ and $B$ are of the same magnification; $C$ through $F$ are of the same magnification. Bar in $B, 10 \mu m$; in $F, 1 \mu m$.

underlying the growth of capillary network found in the present study requires further studies.

Another novel finding in the present study is that alcohol induced remodeling of gap junction in the cardiomyocytes, including up-regulation of the total amount of Cx43 protein (as demonstrated by Western blotting) and redistribution of $\mathrm{Cx} 43$ gap junctions at the cell membrane (confirmed by immunoconfocal microscopy). One might wonder in the present study why the increment of gap junction shown in Western blotting is much less compared to the immunoconfocal results. This is because in the immunoconfocal experiments the expression of gap junctions was calibrated against cell size, which was reduced substantially in the alcohol group. On the other hand, theoretically, more gap junctions in the cardiac muscle should facilitate the electric conduction [25]. In addition, more gap junctions in the lateral borders of cardiomyocytes should attenuate the anisotropism of conduction along the different directions of cardiac muscle and thereby diminish the formation of reentry, a common mechanism underlying the formation of ventricular tachyarrhythmia [26]. However, optical mapping examination in the present study showed that the alcoholtreated mice had a slower conduction and more tachyarrhythmia. In the heart, the propagation of action potential across cardiomyocytes is via gap junctions. Although gap junctions provide low resistance pathways between adjacent cardiomyocytes, the conduction of gap-junctional channels is much slower compared to intracellular conduction. Therefore, when cells became smaller in 

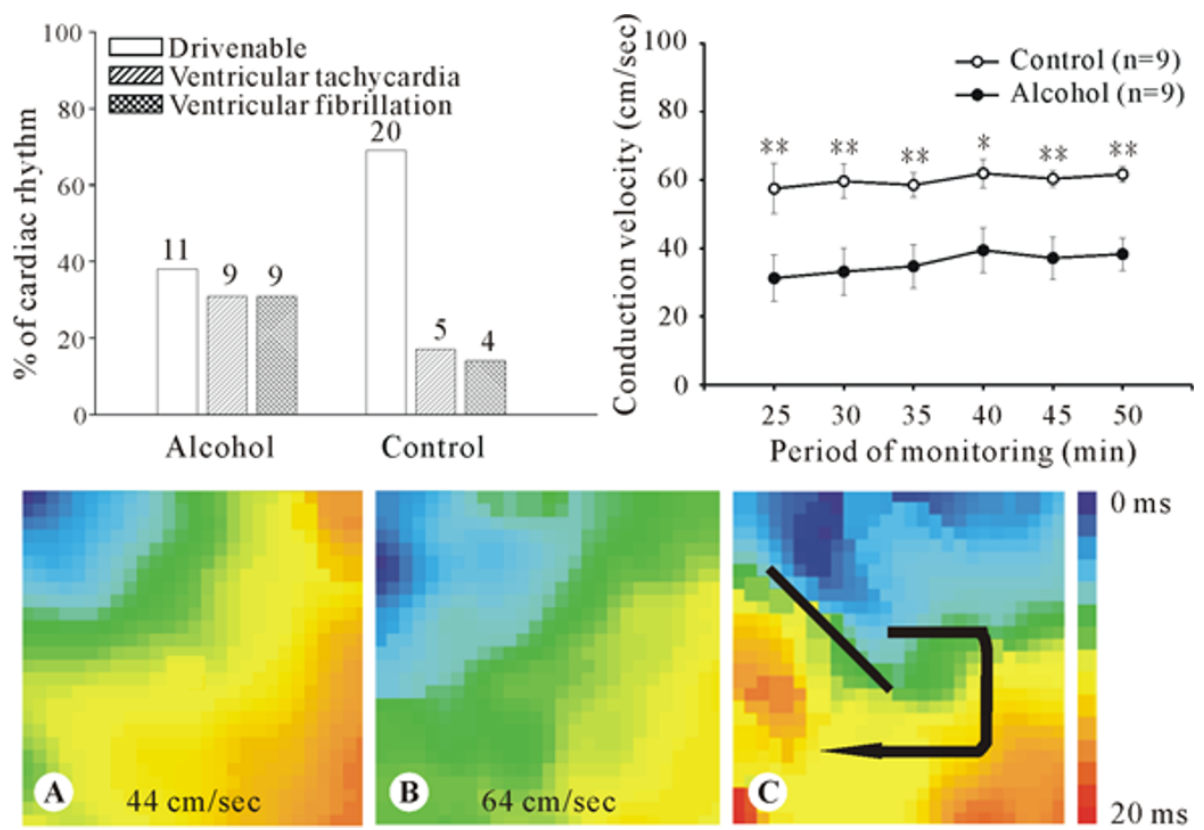

Figure 5 Cardioelectrophysiologic studies of isolated hearts. Upper left histogram shows the percentage of hearts drivenalbe by the pacemaker or manifesting ventricular arrhythmia. The number of animals is indicated at the top of each bar. Upper right histogram shows the values of conduction velocity measured at each time point of both groups. ${ }^{*}, p<0.05$ and ${ }^{* *}, p<0.01$, compared to the control group. Lower panel, examples of activation maps with normal activation sequence from the alcohol (A) and the control group (B), as well as with conduction disturbance $(C)$ from the alcohol group. The black bar means start of the reentry, and the curved arrow indicated the propagation direction Note that the values of conduction velocity in $A$ and $B$ are displayed at the bottom of the maps.

size, for a defined dimension of myocardium, the propagation of action potential must pass more cells and gap junctions in between, and hence, required a longer time. In other words, the conduction became slower [27]. This (the smaller size of cardiomyocytes the slower conduction) suggested that, in the alcohol group of the present study, the change of conduction is attributable to the smaller cell size, which, together with lateralized gap junctions, also affects the anisotropism. Since slow conduction per se also favors the formation of reentry, this may explain that the alcoholic mice were prone to the occurrence of arrhythmia. In this view, increased expression and lateralization of the cardiomyocyte gap junctions may be a protective mechanism of the heart in response to the electric changes. Nevertheless, these findings are in agreement with the clinical observations that cardiac arrhythmias are frequently seen in patients with long-term heavy alcohol use [6].

\section{Conclusions}

In conclusion, chronic 36\% alcohol consumption induced profound remodeling of ventricular myocardium in mice, including change of the size of cardiomyocyte and the expression and distribution of $\mathrm{Cx} 43$ gap junctions, each of which may have distinct contribution to the electrical remodeling, characterized as slow down of conduction and occurrence of tachyarrhythmia.

\section{Methods}

Animals, alcohol administration, and preparation of hearts

The work was conducted in accordance with the Republic of China Animal Protection Law (Scientific Application of Animals), 1998. Eleven-week-old male C57BL/6 mice (purchased from National Laboratory Animal Center, Taipei, Taiwan) were given ad libitum normal chow (Purina 5001, LabDiet ${ }^{\circledR}$, TestDiet company, Indiana, USA) and either double-distilled deionized water or $36 \%$ $\mathrm{v} / \mathrm{v}$ alcohol as their only fluid source for 12 weeks. Thereafter, mice were anesthetized with pentobarbital (i.p., 60 $\mathrm{mg} / \mathrm{kg}$ ). The blood was collected (for determination of alcohol level using Ethanol-L3K Assay (Diagnostic Chemical Limited; Charlottetown, Prince Edward island, Canada)) and the hearts were taken out. The ascending aorta was cannulated and the heart was perfused with standard oxygenated Tyrode's solution $(2.5 \mathrm{ml} / \mathrm{min})$, paced (3-6 Hz, 4 ms duration, 3 fold threshold voltage) at the basal portion of the right ventricle from a stimulus electrode (S88J, GRASS, West Warwick, Rhode Island, USA), and stained with $20 \mu \mathrm{L}$ of di-4-ANEPPS 
(Molecular Probe, Paisley, UK; $2 \mathrm{mM}$ in DMSO). After the cardiac contraction was blocked with cytochalasin D (Sigma, Missouri, USA; $10 \mu \mathrm{M}$ ) the action potentials were recorded using optical mapping. Tiny needles were used to mechanically stabilize the heart. Fifty-eight murine hearts (29 in each group) were used for optical mapping. Conduction velocity, derived from activation map (Figure 5, lower panel), was calculated from the 9 mice of the alcohol group and compared to the initial 9 mice drivenable throughout the recording period of the control group.

In parallel, immediately after removal of the hearts, clearance of blood and weighing, the organs were either stored in liquid nitrogen for Western blotting and immunoconfocal microscopy, or prepared for histological examination and thin-section electron microscopy by standard procedures. In details, 14 hearts (7 in each group) were for Western blotting, 16 hearts ( 8 in each group) for immunohistochemical plus histology examination, and 6 hearts ( 3 in each group) for electron microscopy.

\section{Optical apparatus}

Optical mapping was conducted using a stereo-fluorescence microscopy (Lecia MZ FLIII system; Heidelberg, Germany) with light (100-W high-pressure mercury vapor lamp; 106Z, Lecia) collimated and passed through a $470 \mathrm{~nm}$ interference filter, and focused on the frontal surface of left ventricle. Fluorescence emission from the stained heart was collected with a camera lens $(45 \mathrm{~mm}$, PLAN 1.0×, Lecia) through a 505-nm cut-off filter (0G515, Lecia) and focused to form an image on the surface of a $80 \times 80$ array CCD (CardioCCD39, RedShirtImaging, Georgia, USA). The image was magnified $\times 0.8$ such that the CCD detected a $5 \times 5 \mathrm{~mm}$ area of the epicardium. Spatial and temporal filtering were utilized in data post-processing. Isochronal maps of activation spread and conduction velocity measurements were derived using Cardioplex software (RedShirtImaging).

\section{Immunoconfocal microscopy}

Antibodies and detection systems Rabbit polyclonal antisera (designated Cx43(R530)) against the synthetic peptides corresponding to 314 to 322 of the cytoplasmic $\mathrm{C}$-terminal tail of rat $\mathrm{Cx} 43$ were used. The antisera were affinity-purified and have previously been confirmed to be isotype-specific [28]. Donkey anti-rabbit immunoglobulins conjugated to $\mathrm{CY} 3$ (Chemicon, California, USA) were used to visualize immunolabeled connexin. FITC-conjugated wheat germ agglutinin (WGA; Vector, Burlingame, California, USA) was used to detect the cell border. AntiCD31 (BD Pharmingen, Franklin Lakes, USA) was used to detect the capillary. FITC-conjugated anti-rat immunoglobulins (Molecular Probes, Paisley, UK) were used to visualize immunolabeled capillary [29].

Immunolabeling Cryosections of the heart were fixed in $-20 * \mathrm{C}$ methanol, rinsed in PBS for 5 minutes, blocked in $0.5 \%$ BSA (15 minutes), and incubated at $37{ }^{*} \mathrm{C}$ with antiCx43 (1:300) for 1 hour or with anti-CD31 (1:200) for 2 hours. The samples were then treated with CY3-conjugated secondary antibody (1:500, room temperature, 1 hour). For single labelling of WGA, incubation was with WGA-FITC $\left(1: 500,37^{*} \mathrm{C}, 1\right.$ hour). In double labeling experiments, incubation was with a mixture of antiCx43 (1:300) plus WGA-FITC (1:500) at 37*C for 1 hour, followed by incubation with the CY3-conjugated secondary antibody.

Confocal laser scanning microscopy Immunostained samples were examined by confocal laser scanning microscopy using a Leica TCS SP equipped with argon/krypton laser. Single WGA-FITC-labeled samples were used to measure the size of cardiomyocytes. For double labeling of Cx43 and WGA, the images were taken using simultaneous dual channel scanning. The images were collected using the $\times 40$ objective lens and zoom 1.0 computer setting so that each pixel represented $0.23 \mu \mathrm{m}$. Each image recorded consisted of $1024 \times 1024$ pixels, and projection views of 4 consecutive optical sections taken at $1 \mu \mathrm{m}$ intervals in the middle of the sections were recorded for analysis.

\section{Western blotting}

Tissue samples of left ventricular free wall, ground to fine powders under liquid nitrogen, were lysed with $0.5 \mathrm{ml}$ SB20 (containing 20\% SDS, $0.1 \mathrm{M}$ Tris $\mathrm{pH} 6.8$, and $10 \mathrm{mM}$ ETDA) and homogenized by sonication. After protein estimation, 2.5\% 2-mercaptoethanol was added to the remaining samples. SDS-polyacrylamide gel electrophoresis was performed using minigels made of $4.5 \%$ stacking gels and $10 \%$ separation gels. Twenty $\mu \mathrm{g}$ of each sample, mixed with sample buffer (SB20 containing 2.5\% 2 -mercaptoethanol and $1 \%$ bromophenol blue) to make the final volume $15 \mu \mathrm{l}$, was loaded in each lane, subjected to electrophoresis ( $60 \mathrm{~V}$ for running in stacking gel and $120 \mathrm{~V}$ in separation gel; constant voltage), and transferred ( $20 \mathrm{~V}$, constant voltage at room temperature overnight) onto PVDF membrane (Perkin Elmer, USA). The membrane was probed for $\mathrm{Cx} 43$ (1:200) and visualized using CDP-Star substrate solution (Roche, USA). Finally the membranes were stripped with $2 \%$ SDS, $0.7 \%$ 2-mercaptoethanol, and $0.1 \mathrm{M}$ Tris $\mathrm{pH} 6.8$, and probed with a mouse anti- $\beta$-actin antibody (1:5000; Chemicon).

\section{Analysis and statistics}

Image analysis was conducted using QWIN image analysis software (Leica). The amount of capillary network was 
expressed as the number of capillary per unit area. For myocyte size and $\mathrm{Cx} 43$ gap junction distribution, images taken from the epicardial and endocardial portions of the sections at the transverse plane of left ventricle were used. The following data were obtained for each group: i) the length, width, and area of individual ventricular myocyte; ii) the total area of immunolabeled gap junctions per myocyte area, expressed in percentage; and iii) the percentage of immunolabeled gap junctions along the lateral borders of individual myocytes. For comparison of myocardial cellularity, cryosections stained with haematoxylin were recorded using a digital camera. The nuclei were counted and expressed as the number per unit area. For Western blotting, densitometric scanning and analysis were performed on the blots using Imagemaster (Amersham Pharmacia Biotech, New Jersey, USA). Results were expressed as mean \pm SE. The data of histological examination and Western blotting were compared by Student's t test. For conduction velocity, data were compared by two way ANOVA with repeat measurement. Incidence of ventricular tachycardia, fibrillation were compared statistically by Chi square.

\section{Acknowledgements}

Supported by grants NSC 97-2314-B-715-003-MY3 from the National Science Council, Taiwan and MMH 9943 and MMH-E 97003 from the Medical Research Department of the Mackay Memorial Hospital, Taiwan.

\section{Author details}

'Departments of Internal Medicine and Medical Research, Mackay Memorial Hospital, Mackay Medicine, Nursing and Management College, Mackay Medical College, New Taipei City, Taiwan. ${ }^{2}$ Departments of Physiology and Biophysics, National Defense Medical Center, Taipei, Taiwan. ${ }^{3}$ The First Cardiovascular Division, Department of Internal Medicine, Chang Gung Memorial Hospital, Taipei, Taiwan.

\section{Authors' contributions}

YJ carried out the cardioelectrophysiologic studies, participated in the cardiac conduction, arrhythmia research and drafted the manuscript. $\mathrm{CL}$ carried out the morphometrical analysis of the study. RC participated in the electron microscopy. YM participated in the protein analysis (western blot and immunostaining). YS carried out the interpretation of the findings. Cl participated in the design of the study and performed the statistical analysis. $\mathrm{CH}$ participated in the design of the study and critical comments of the manuscript. $\mathrm{HI}$ conceived of the study, and participated in its design and coordination and helped to draft the manuscript. All authors read and approved the final manuscript.

\section{Competing interests}

The authors declare that they have no competing interests.

Received: 11 July 2011 Accepted: 29 September 2011 Published: 29 September 2011

\section{References}

1. Lang CH, Frost RA, Summer AD, Vary TC: Molecular mechanisms responsible for alcohol-induced myopathy in skeletal muscle and heart. Int J Biochem Cell Biol 2005, 37:2180-2195.

2. Lee WK, Regan TJ: Alcoholic cardiomyopathy: is it dose-dependent? Congest Heart Fail 2002, 8:303-306.

3. Patel VB, Why HJ, Richardson PJ, Preedy VR: The effects of alcohol on the heart. Adverse Drug React Toxicol Rev 1997, 16:15-43.
4. lacovoni A, De Maria R, Gavazzi A: Alcoholic cardiomyopathy. J Cardiovasc Med (Hagerstown) 2010, 11:884-892.

5. Goodkind MJ, Gerber NH, Mellen JR, Kostis JB: Altered intracardiac conduction after acute administration of ethanol in the dog. $J$ Pharmacol Exp Ther 1975, 194:633-638.

6. Kupari M, Koskinen P: Alcohol, cardiac arrhythmias and sudden death. Novartis Found Symp 1998, 216:68-79, discussion 79-85.

7. Williams ES, Mirro MJ, Bailey JC: Electrophysiological effects of ethanol, acetaldehyde, and acetate on cardiac tissues from dog and guinea pig. Circ Res 1980, 47:473-478.

8. Gros DB, Jongsma HJ: Connexins in mammalian heart function. Bioessays 1996, 18:719-730.

9. Severs NJ, Coppen SR, Dupont E, Yeh HI, Ko YS, Matsushita T: Gap junction alterations in human cardiac disease. Cardiovasc Res 2004, 62:368-377.

10. Gutstein DE, Morley GE, Vaidya D, Liu F, Chen FL, Stuhlmann H, Fishman Gl: Heterogeneous expression of Gap junction channels in the heart leads to conduction defects and ventricular dysfunction. Circulation 2001, 104:1194-1199.

11. Lerner DL, Yamada KA, Schuessler RB, Saffitz JE: Accelerated onset and increased incidence of ventricular arrhythmias induced by ischemia in Cx43-deficient mice. Circulation 2000, 101:547-552.

12. Rhodes JS, Best K, Belknap JK, Finn DA, Crabbe JC: Evaluation of a simple model of ethanol drinking to intoxication in C57BL/6J mice. Physiol Behav 2005, 84:53-63.

13. Piano MR: Alcoholic cardiomyopathy: incidence, clinical characteristics, and pathophysiology. Chest 2002, 121:1638-1650.

14. Kupari M, Koskinen P, Suokas A: Left ventricular size, mass and function in relation to the duration and quantity of heavy drinking in alcoholics. Am J Cardiol 1991, 67:274-279.

15. Lazarevic AM, Nakatani S, Neskovic AN, Marinkovic J, Yasumura Y, Stojicic D, Miyatake K, Bojic M, Popovic AD: Early changes in left ventricular function in chronic asymptomatic alcoholics: relation to the duration of heavy drinking. J Am Coll Cardiol 2000, 35:1599-1606.

16. Urbano-Marquez A, Fernandez-Sola J: Alcohol consumption and heart failure. $J$ Card Fail 2005, 11:329-332.

17. Urbano-Marquez A, Estruch R, Navarro-Lopez F, Grau JM, Mont L, Rubin E: The effects of alcoholism on skeletal and cardiac muscle. N Engl J Med 1989, 320:409-415.

18. Wang L, Zhou Z, Saari JT, Kang YJ: Alcohol-induced myocardial fibrosis in metallothionein-null mice: prevention by zinc supplementation. Am J Pathol 2005, 167:337-344.

19. Preedy VR, Reilly ME, Patel VB, Richardson PJ, Peters TJ: Protein metabolism in alcoholism: effects on specific tissues and the whole body. Nutrition 1999, 15:604-608.

20. Mall G, Mattfeldt T, Rieger P, Volk B, Frolov VA: Morphometric analysis of the rabbit myocardium after chronic ethanol feeding-early capillary changes. Basic Res Cardiol 1982, 77:57-67.

21. Vikhert AM, Tsiplenkova VG, Cherpachenko NM: Alcoholic cardiomyopathy and sudden cardiac death. J Am Coll Cardiol 1986, 8:3A-11A.

22. Gu JW, Elam J, Sartin A, Li W, Roach R, Adair TH: Moderate levels of ethanol induce expression of vascular endothelial growth factor and stimulate angiogenesis. Am J Physiol Regul Integr Comp Physiol 2001, 281: R365-372.

23. Jankala H, Eriksson PC, Eklund K, Sarviharju M, Harkonen M, Maki T: Effect of chronic ethanol ingestion and gender on heart left ventricular p53 gene expression. Alcohol Clin Exp Res 2005, 29:1368-1373.

24. Teodoro JG, Evans SK, Green MR: Inhibition of tumor angiogenesis by p53: a new role for the guardian of the genome. J Mol Med 2007, 85:1175-1186

25. Spach MS, Starmer CF: Altering the topology of gap junctions a major therapeutic target for atrial fibrillation. Cardiovasc Res 1995, 30:337-344.

26. Saffitz JE, Davis LM, Darrow BJ, Kanter HL, Laing JG, Beyer EC: The molecular basis of anisotropy: role of gap junctions. J Cardiovasc Electrophysiol 1995, 6:498-510.

27. Spach MS, Heidlage JF, Dolber PC, Barr RC: Electrophysiological effects of remodeling cardiac gap junctions and cell size: experimental and model studies of normal cardiac growth. Circulation Research 2000, 86:302-311.

28. Yeh HI, Lai YJ, Lee YN, Chen YJ, Chen YC, Chen CC, Chen SA, Lin Cl, Tsai CH: Differential expression of connexin43 gap junctions in cardiomyocytes isolated from canine thoracic veins. J Histochem Cytochem 2003, 51:259-266. 
29. Jacobi J, Tam BY, Wu G, Hoffman J, Cooke JP, Kuo CJ: Adenoviral gene transfer with soluble vascular endothelial growth factor receptors impairs angiogenesis and perfusion in a murine model of hindlimb ischemia. Circulation 2004, 110:2424-2429.

doi:10.1186/1423-0127-18-72

Cite this article as: Lai et al:: Slow conduction and gap junction

remodeling in murine ventricle after chronic alcohol ingestion. Journal of Biomedical Science 2011 18:72.

Submit your next manuscript to BioMed Central and take full advantage of:

- Convenient online submission

- Thorough peer review

- No space constraints or color figure charges

- Immediate publication on acceptance

- Inclusion in PubMed, CAS, Scopus and Google Scholar

- Research which is freely available for redistribution

Submit your manuscript at www.biomedcentral.com/submit 